PROCEEDINGS OF THE AMERICAN MATHEMATICAL SOCIETY

Volume 127, Number 7, Pages 1917-1921

S 0002-9939(99)04760-7

Article electronically published on February 17, 1999

\title{
COMPOSITION SERIES OF MODULES OVER PRÜFER DOMAINS
}

\author{
BRUCE OLBERDING
}

(Communicated by Wolmer V. Vasconcelos)

\begin{abstract}
A weakened version of the Jordan-Hölder theorem is shown to hold for torsion-free finite rank modules over an integral domain $R$ precisely when $R$ is a Prüfer domain.
\end{abstract}

\section{INTRODUCTION}

If $R$ is an integral domain, a composition series ${ }^{1}$ of a torsion-free $R$-module $G$ of finite rank $n$ is a sequence $\left\{G_{i}\right\}_{i=1}^{n}$ of distinct submodules of $G$ such that $0 \neq G_{1} \subset G_{2} \subset \ldots \subset G_{n}=G$ and $G_{i+1} / G_{i}$ is torsion-free for all $i=1,2, \ldots, n-1$ [10]. The chief factors, $F_{i}$, of the sequence $\left\{G_{i}\right\}_{i=1}^{n}$ are defined by $F_{1}=G_{1}$ and $F_{i+1}=G_{i+1} / G_{i}$ for all $i=1,2, \ldots, n-1$. It is not difficult to show that for each torsion-free finite rank module $G$ there exists a composition series of $G$.

In general, the chief factors of a composition series are not unique up to isomorphism. Even when $R$ is a PID, a Jordan-Hölder theorem for the composition series of a module is the exception rather than the rule [10, Lemma 4]. However, in [8] (but see also [9]) O. Mutzbauer showed that for torsion-free finite rank abelian groups the tensor product of the chief factors is in fact unique up to isomorphism, and thus is an invariant for a torsion-free abelian group. ${ }^{2}$

Mutzbauer's proof relied on particular decomposition properties of torsion modules that are specific to principal ideal domains and not available in more general settings. ${ }^{3}$ In this paper we present a different approach to his result. Using the theory of exterior powers we are able to avoid arguments involving torsion modules and to extend Mutzbauer's result to Prüfer domains. It is shown moreover that the invariance of the tensor product of chief factors characterizes the class of Prüfer domains. In the course of proving this, we establish an interpetation of the exterior

Received by the editors April 12, 1997 and, in revised form, September 24, 1997.

1991 Mathematics Subject Classification. Primary 13F05, 13C05; Secondary 15A75, 20 K15.

Key words and phrases. Prüfer domain, torsion-free module, exterior algebra.

Some of these results appeared in the author's Ph.D. dissertation, which was written under the supervision of Professor J. D. Reid at Wesleyan University.

${ }^{1}$ Also known as "composition sequence" [8] and "composition chain" [9]. It can be checked that if $R$ is a valuation domain, our definition coincides with the notion of a pure composition series defined in [3].

${ }^{2}$ The rank 2 case for abelian groups was first proved by R. Beaumont and R. Pierce [1, Lemma 4.13].

${ }^{3}$ Mutzbauer did prove a weaker version of his result for torsion-free finite rank modules over Dedekind domains, namely, that the tensor product of chief factors is unique up to quasiisomorphism. 
power of a finite rank torsion-free module $G$ over a Prüfer domain in terms of the composition series of $G$. Other characterizations of Prüfer domains are noted in the process.

The author wishes to thank the referee for several helpful suggestions and corrections. In particular, the elegant approach to Proposition 2.2 via Lemma 2.1 is due to the referee.

By integral domain we mean a commutative ring with identity having no zero divisors. If $R$ is an integral domain, then $Q$ will designate the quotient field of $R$. A Prüfer domain $R$ is an integral domain for which every finitely generated ideal is invertible; equivalently, $R_{M}$ is a valuation domain for every maximal ideal $M$ of $R$.

Let $R$ be an integral domain with quotient field $Q$ and let $G$ be a torsionfree module. The unadorned symbol $\otimes$ will stand for $\otimes_{R}$. The rank of $G$ is the dimension of the $Q$-vector space, $Q \otimes G$; equivalently, the rank of $G$ is the cardinality of a maximal linearly independent subset of $G$. If, in addition, $R$ is a Prüfer domain, then a pure submodule of $G$ is a submodule $H$ of $G$ such that $G / H$ is torsion-free $\left[3\right.$, Theorem 4.5]. Note that in this case $\left\{G_{i}\right\}_{i=1}^{n}$ is a composition series of a torsionfree module $G$ of rank $n$ if and only if $\left\{G_{i}\right\}_{i=1}^{n}$ is an ascending chain of distinct nontrivial pure submodules of $G$.

\section{COMPOSITION SERIES AND EXTERIOR POWERS OF MODUlES OVER PRÜFER DOMAINS}

Recall from the introduction the definition of a composition series of a torsionfree module. In this section we establish that an integral domain $R$ is a Prüfer domain if and only if, for each torsion-free finite rank module $G$, the tensor product of chief factors of a composition series for $G$ is invariant (up to isomorphism) under the choice of composition series for $G$. Since our approach involves exterior powers, we note first a few useful properties of this construction. The reader is referred to [2, Chapter III, Sections 2-5] for other basic results involving exterior powers.

Lemma 2.1. If $R$ is an integral domain and $G$ is a flat $R$-module, then $\bigwedge G$ is a torsion-free $R$-module.

Proof. A module is flat if and only if it is a direct limit of projective modules [7]. Since the operator $\bigwedge$ commutes with direct limits [2, Chapter III, Section 7.6, Proposition 6], it follows that for a flat $R$-module $G, \wedge G$ is a direct limit of projective modules [2, Chapter III, Section 7.8, Corollary 2] and hence is a flat $R$-module.

Proposition 2.2. An integral domain $R$ is a Prüfer domain if and only if $\wedge^{k} G$ is a torsion-free $R$-module for all $k>0$ and torsion-free $R$-modules $G$.

Proof. Assume $\wedge^{k} G$ is torsion-free for all $k>0$ and torsion-free $R$-modules $G$. To prove that $R$ is a Prüfer domain, it suffices to show that $G \otimes I$ is torsion-free for all ideals $I$ of $R$ and torsion-free modules $G$ [6, Theorem 2]. Let $G$ be a torsion-free module. If $I$ is an ideal of $R$, then $G \otimes I$ is a summand of $\bigwedge^{2}(G \oplus I)$ [2, Chapter III, Section 7]. Thus by assumption $G \otimes I$ is torsion-free. The converse follows from Lemma 2.1 and the fact that torsion-free modules over Prüfer domains are flat [11, Theorem 4.33]. 
Corollary 2.3. Let $G$ be a torsion-free module over a Prüfer domain $R$ and let $g_{1}, g_{2}, \ldots, g_{k}$ be distinct elements of $G$. Then $\left\{g_{1}, g_{2}, \ldots, g_{k}\right\}$ is a linearly independent subset of $G$ if and only if $g_{1} \wedge g_{2} \wedge \cdots \wedge g_{k} \neq 0$.

Proof. If $R$ is an integral domain and $G$ is a torsion-free $R$-module, then $\left\{g_{1}, g_{2}, \ldots\right.$, $\left.g_{n}\right\}$ is a linearly dependent subset of $G$ if and only if there exists a non-zero element $r$ of $R$ such that $r g_{1} \wedge g_{2} \wedge \cdots \wedge g_{n}=0$ [4, Proposition 12.3]. The claim follows now from Proposition 2.2.

Corollary 2.4. If $R$ is a Prüfer domain and $G$ is a torsion-free $R$-module of finite rank $n$, then $\wedge^{k} G$ is a torsion-free $R$-module of rank $\left(\begin{array}{c}n \\ k\end{array}\right)$ for each positive integer number $k$.

The following terminology will be helpful in stating and proving Proposition 2.6. Let $R$ be an integral domain and let $G$ be an $R$-module. If $k, l>0, A$ is a submodule of $\wedge^{k} G$ and $B$ is a submodule of $\bigwedge^{l} G$, then $A \wedge B$ is defined to be the submodule of $\bigwedge^{k+l} G$ generated by the set $\{a \wedge b \in \wedge G: a \in A, b \in B\}$. It can be verified that $A \wedge B=B \wedge A$, and that if $M \in \operatorname{Max}(R)$, then $(A \wedge B)_{M}=A_{M} \wedge B_{M}$. Furthermore, if $C$ is a submodule of $\wedge^{m} G$ for some $m>0$, then $A \wedge(B \wedge C)=(A \wedge B) \wedge C$.

Lemma 2.5. Let $R$ be a Prüfer domain and let $G$ be a torsion-free finite rank $R$ module. If $H$ is a submodule of $G$, then $\bigwedge^{k} H$ can be identified with its image in $\wedge^{k} G$ for all positive integers $k$.

Proof. Let $R$ be a Prüfer domain, let $H$ be a submodule of a torsion-free finite rank module $G$, and let $k$ be some positive integer. Let $n$ denote the rank of $H$. If $k>n$, then $\bigwedge^{k} H=0$ (Corollary 2.4), so we may assume that $k \leq n$. Choose a maximal linearly independent subset $\left\{h_{1}, h_{2}, \ldots, h_{n}\right\}$ of $H$, and let $\mathcal{J}=\{J \subset$ $\{1,2, \ldots, n\}: J$ has $k$ elements $\}$. For each $J \in \mathcal{J}$, define $h_{J}=h_{j_{1}} \wedge h_{j_{2}} \wedge \cdots \wedge h_{j_{k}}$, where $J=\left\{j_{1}, j_{2}, \ldots, j_{k}\right\}$. Then $\left\{h_{J}: J \in \mathcal{J}\right\}$ is a maximal linearly independent set of elements of $\bigwedge^{k} H$. Define $\alpha$ to be the canonical homomorphism of $\bigwedge^{k} H$ into $\bigwedge^{k} G$. Suppose now that $x \in \bigwedge^{k} H$ and that $\alpha(x)=0$ in $\bigwedge^{k} G$. There exists for each $J \in \mathcal{J}$ an element $r_{J} \in R$ such that $r x=\sum_{J \in \mathcal{J}} r_{J} h_{J}$, for some non-zero element $r \in R$. Since $\alpha(r x)=0$, it must be the case that $\sum_{J \in \mathcal{J}} r_{J} \alpha\left(h_{J}\right)=0$. But $\left\{h_{1}, h_{2}, \ldots, h_{n}\right\}$ is a linearly independent subset of $G$ and for all $J, J^{\prime} \in \mathcal{J}$, $\alpha\left(h_{J}\right)=\alpha\left(h_{J^{\prime}}\right)$ if and only if $J=J^{\prime}$. Hence $\left\{\alpha\left(h_{J}\right): J \in \mathcal{J}\right\}$ is a linearly independent subset of $\bigwedge^{k} G$ and $r_{J}=0$ for all $J \in \mathcal{J}$. It follows that $r x=0$. By Proposition 2.2, $x=0$.

Proposition 2.6. Let $R$ be a Prüfer domain and let $G$ be a torsion-free $R$-module of finite rank $n$. If $\left\{G_{i}\right\}_{i=1}^{n}$ is a composition series for $G$ and $k \leq n$, then $\wedge^{k} G=$ $G_{n} \wedge G_{n-1} \wedge \ldots \wedge G_{n-k+1}$.

Proof. It suffices to check the result locally. Therefore we may assume that $R$ is a valuation domain. If the rank of $G$ is one, then the proposition is clearly true, so suppose that $G$ has rank greater than one. We begin by showing that $\wedge^{2} G=G_{n} \wedge G_{n-1}$ and remark that it is enough to show $u \wedge v \in G_{n} \wedge G_{n-1}$ for all $u, v \in G$. Let $u \wedge v$ be a non-zero element of $\wedge^{2} G$. If either $u$ or $v$ is in $G_{n-1}$, then there is nothing to prove, so suppose that $u, v \notin G_{n-1}$. Since $G / G_{n-1}$ is a torsion-free $R$-module of rank one, there exist non-zero elements $r$ and $s$ in $R$ so that $r u+s v \in G_{n-1}$. Furthermore, $r u+s v \neq 0$, since $u \wedge v \neq 0$ 
(Corollary 2.3). Because $\mathrm{R}$ is a valuation domain, either $r$ divides $s$ or $s$ divides $r$. Without loss of generality, assume that $r=t s$ for some non-zero element $t$ of $R$. We have now $r u+s v=t s u+s v=s(t u+v) \in G_{n-1}$. Since $G / G_{n-1}$ is torsion-free, it must be the case that $t u+v=g$ for some $g \in G_{n-1}$. Thus $u \wedge v=u \wedge(g-t u)=u \wedge g-u \wedge t u=u \wedge g \in G_{n} \wedge G_{n-1}$.

An induction argument finishes the proof. Assume that the proposition holds for all torsion-free $R$-modules of rank $m$ where $m<n$. By Lemma $2.5, \wedge^{k-1} G_{n-1}$ can be identified with its image in $\wedge^{k-1} G$, i.e., with $G_{n-1} \wedge G_{n-1} \wedge \cdots \wedge G_{n-1}$ $(k-1$ copies $)$. Thus $\wedge^{k} G=\left(\wedge^{k-2} G\right) \wedge\left(\bigwedge^{2} G\right)=\left(\wedge^{k-2} G\right) \wedge G \wedge G_{n-1}=$ $\left(\wedge^{k-3} G\right) \wedge\left(\bigwedge^{2} G\right) \wedge G_{n-1}=\left(\bigwedge^{k-3} G\right) \wedge G \wedge G_{n-1} \wedge G_{n-1}=\left(\wedge^{k-4} G\right) \wedge G \wedge$ $G_{n-1} \wedge G_{n-1} \wedge G_{n-1}=\ldots=G \wedge\left(\wedge^{k-1} G_{n-1}\right)$. By the induction hypothesis, $\wedge^{k-1} G_{n-1}=G_{n-1} \wedge G_{n-2} \wedge \ldots \wedge G_{n-k+1}$.

If $R$ is a Prüfer domain and $G$ is a torsion-free $R$-module of finite rank $n$, then the torsion-free rank one module $\bigwedge^{n} G$ can be interpreted in light of Proposition 2.6. The result of Mutzbauer discussed in the introduction can be derived for Prüfer domains by making use of the rank one module $\bigwedge^{n} G$, which it will be helpful to denote as $\bigwedge^{*} G$.

Proposition 2.7. Let $R$ be a Prüfer domain and let $G$ be a torsion-free finite rank $R$-module. If $\left\{G_{i}\right\}_{i=1}^{n}$ is a composition series for $G$ with chief factors $\left\{F_{i}\right\}_{i=1}^{n}$, then

$$
\bigwedge^{*} G \cong F_{1} \otimes F_{2} \otimes \ldots \otimes F_{n} .
$$

Proof. Let $\left\{G_{i}\right\}_{i=1}^{n}$ be a composition series for $G$ with chief factors $\left\{F_{i}\right\}_{i=1}^{n}$. Define a homomorphism $\rho: G_{1} \otimes G_{2} \otimes \cdots \otimes G_{n} \mapsto \wedge^{*} G$ by $\rho\left(g_{1} \otimes g_{2} \cdots \otimes g_{n}\right)=g_{1} \wedge g_{2} \wedge \cdots \wedge g_{n}$, where $g_{i} \in G_{i}$ for each $i=1,2, \ldots, n$. Note that by Proposition 2.6, $\rho$ is a surjection. Let $K=K e r: \rho$. We claim that $K$ is generated by all elements of the form $g_{1} \otimes g_{2} \otimes \cdots \otimes g_{n}$, where $g_{i} \in G_{i}$ for $i=1,2, \ldots, n$ and $g_{i}=g_{j}$ for some $i \neq j$ or $\left\{g_{1}, g_{2}, \ldots, g_{n}\right\}$ is a linearly dependent subset of $G$. By Corollary $2.3, K \subseteq K e r: \rho$. To prove Ker: $\rho \subseteq K$, it suffices to argue locally, in which case we can assume that $R$ is a valuation domain. Let $x \in K e r: \rho$ and write $x=\sum_{j=1}^{l} x_{j}$, where for each $j, x_{j}=g_{j 1} \otimes g_{j 2} \otimes \cdots \otimes g_{j n} \in G_{1} \otimes G_{2} \otimes \cdots \otimes G_{n}$. Then $0=\rho(x)=\sum_{j=1}^{l} \rho\left(x_{j}\right)$. Given any two non-zero elements $r, s \in R$, either $r$ divides $s$ or $s$ divides $r$. This and the fact that $\Lambda^{*} G$ is a torsion-free module of rank one implies that we can assume without loss of generality that for each $j \neq 1$, there exists $r_{j} \in R$ such that $r_{j} x_{1}=x_{j}$. Define $r=1+r_{2}+r_{3}+\cdots+r_{l}$. Then $x=r x_{1}$, and so by Proposition $2.2 \rho\left(x_{1}\right)=0$, which implies by Corollary 2.3 that either $g_{1 i}=g_{1 j}$ for some $i \neq j$ or $\left\{g_{11}, g_{12}, \ldots, g_{1 n}\right\}$ is a linearly dependent subset of $G$. Therefore, $x_{1} \in K$. It follows that $x \in K$, and the claim is proved. Using a similar reduction to the local case, it can be checked that $K$ is also the kernel of the canonical surjection $G_{1} \otimes G_{2} \otimes \cdots \otimes G_{n} \mapsto F_{1} \otimes F_{2} \otimes \cdots \otimes F_{n}$. This completes the proof.

We can now prove the main theorem.

Theorem 2.8. An integral domain $R$ is a Prüfer domain if and only if, for each torsion-free finite rank module $G$, the tensor product of chief factors of a composition series for $G$ is invariant (up to isomorphism) under the choice of composition series for $G$.

Proof. If $R$ is a Prüfer domain, then sufficiency follows from Proposition 2.7. To prove the converse, it is enough to show that every ideal generated by two elements 
is invertible [5, Theorem 22.1]. Let $I$ be an ideal of $R$ generated by two elements of $I$. There is an exact sequence $0 \rightarrow K \rightarrow G \rightarrow I \rightarrow 0$, where $G$ is a rank two free $R$-module and $K$ is a pure submodule of $G$ isomorphic to a fractional ideal of $R$. Since $G$ is a free module, we may choose a composition series $\left\{G_{1}, G_{2}\right\}$ for $G$ consisting of free submodules of $G$. By assumption $I \otimes K \cong G_{1} \otimes\left(G_{2} / G_{1}\right)$. Observe that $G_{1} \otimes\left(G_{2} / G_{1}\right)$ (and hence $I \otimes K$ ) is a free module of rank one, thus proving necessity.

Corollary 2.9. An integral domain $R$ is a Prüfer domain if and only if for each exact sequence $0 \rightarrow A \rightarrow G \rightarrow B \rightarrow 0$ of torsion-free finite rank $R$-modules, $\bigwedge^{*} G \cong \bigwedge^{*} A \otimes \bigwedge^{*} B$

Proof. Suppose first that $R$ is a Prüfer domain. Without loss of generality, assume that $A$ is a pure submodule of $G$. Let $\left\{A_{i}\right\}_{i=1}^{n}$ be a composition series of $A$, and extend this to a composition series of $G,\left\{A_{1}, A_{2}, \ldots, A_{n}, B_{1}, B_{2}, \ldots, B_{m}\right\}$. Observe that $\left\{B_{1} / A, B_{2} / A, \ldots, B_{m} / A\right\}$ is a composition series for $G / A \cong B$. By Theorem 2.7, $\bigwedge^{*} A \cong A_{1} \otimes A_{2} / A_{1} \otimes \cdots \otimes A_{n} / A_{n-1}$. Since $\left(B_{j} / A\right) /\left(B_{j-1} / A\right) \cong$ $B_{j} / B_{j-1}$, it follows also that $\bigwedge^{*} G / A \cong B_{1} / A \otimes B_{2} / B_{1} \otimes \cdots \otimes B_{m} / B_{m-1}$. Thus $\Lambda^{*} G \cong \Lambda^{*} A \otimes \Lambda^{*} B$.

Conversely, if $I$ is an ideal of $R$ generated by two elements, then there is an exact sequence $0 \rightarrow K \rightarrow G \rightarrow I \rightarrow 0$, where $G$ is a rank two free $R$-module and $K$ is a pure submodule of $G$ isomorphic to a fractional ideal of $R$. By assumption, $\bigwedge^{*} G \cong K \otimes I$. Also, $\bigwedge^{*} G$ is a free module of rank one. Thus $I$ is an invertible ideal of $R$ and $R$ must be a Prüfer domain.

\section{REFERENCES}

[1] R. A. Beaumont and R. S. Pierce, Torsion-free groups of rank two, Mem. Amer. Math. Soc. 72 (1961). MR 24:A162

[2] N. Bourbaki, Elements of mathematics: Algebra I, Chapters 1 - 3, Springer-Verlag, 1989. MR 90d:00002

[3] L. Fuchs and L. Salce, Modules over valuation domains, Lecture notes in pure and applied mathematics 96, Marcel Dekker, New York, 1985. MR 86h:13008

[4] R. B. Gardner, Exterior algebras over commutative rings, University of North Carolina at Chapel Hill, 1972. MR 53:10839

[5] R. W. Gilmer, Multiplicative Ideal Theory, Queen's Papers in Pure and Applied Mathematics 90, Queen's University, 1992. MR 93j:13001

[6] A. Hattori, On Prüfer rings, J. Math. Soc. Japan 9 (1957), 381-385. MR 20:854b

[7] D. Lazard, Autour de la platitude, Bull. Soc. Math. France 97 (1969), 81-128. MR 40:7310

[8] O. Mutzbauer, Type graph, Springer-Verlag Lecture Notes 1006, 228-252. MR 85d:20052

[9] O. Mutzbauer, Type invariants of torsion-free abelian groups, Contemporary Math. 87 (1989), 133-154. MR 90c:20069

[10] J. J. Rotman, The Grothendieck group of torsion-free abelian groups of finite rank, Proc. London Math. Soc. (3) 13 (1963), 724-732. MR 27:4857

[11] J. J. Rotman, An introduction to homological algebra, Academic Press, 1979.

Department of Mathematics, Northeast Louisiana University, Monroe, Louisiana 71209

E-mail address: maolberding@alpha.nlu.edu 